\title{
Augmentative and Alternative Communication for Speaking Autistic Adults: Overview and Recommendations
}

\author{
Alyssa Hillary Zisk, MS, ${ }^{1}$ and Elizabeth Dalton, $\mathrm{PhD}^{2}$
}

\begin{abstract}
In recent years, technologies used for augmentative and alternative communication (AAC) have seen increasing development and availability. As a result, more and more autistic people are using AAC. With the increased use of AAC by autistic people, research on autism and AAC has also increased. However, the vast majority of this research focuses on nonspeaking autistic children. AAC use by autistic adults and by speaking autistic people has received limited academic attention. Speaking autistic adults often use AAC and many have publicly shared information about their strategies and experiences. In this article, we provide an overview of the speech differences autistic adults choose to support through AAC, including varying difficulties with speech depending on environment and content. We also discuss the technologies and strategies adopted by autistic adults, including free or low-cost tools that are not specific to AAC, mobile technologies, and signed languages or gestures. We explore barriers to AAC use, including a lack of awareness of relevant options, misconceptions about who AAC supports are for, and the cost of dedicated AAC applications or devices. We then provide suggestions for autistic adults, people supporting autistic adults, and researchers. Overall communicationrather than speech-should be prioritized. Mainstream communication technologies can support communication for autistic adults and a variety of tools can support communication across contexts. Further research into the use and effectiveness of AAC for autistic adults is needed, as is research on barriers to AAC use.
\end{abstract}

Keywords: augmentative and alternative communication, communication support, autistic adults

\section{Lay Summary}

What is augmentative and alternative communication?

Augmentative and alternative communication (AAC) describes the ways people communicate without, or in addition to, speech.

What do we know about AAC for autistic adults who can speak?

Research on AAC and autism has focused on nonspeaking children. However, autistic adults who use AAC sometimes tell each other about AAC. This community knowledge includes reasons AAC is important, useful AAC strategies, and barriers to AAC use. This article talks about autistic community knowledge about AAC and then makes suggestions.

Why is AAC important for autistic adults who can speak?

Autistic adults, including those who talk, may not always be able to meet all their communication needs with speech alone. Autistic people who use speech may experience intermittent, unreliable, and/or insufficient speech.

What AAC strategies do speaking autistic adults use?

The three main ways that autistic adults report on using AAC include:

(1) Free or low-cost tools that are not specific to AAC (e.g., online chat rooms, text messaging applications, or handwriting).

\footnotetext{
${ }^{1}$ Interdisciplinary Neuroscience Program, University of Rhode Island, Kingston, Rhode Island.

${ }^{2}$ Communication Disorders, University of Rhode Island, Kingston, Rhode Island.
} 
(2) Mobile applications designed for communication support.

(3) Signed languages such as American Sign Language.

What are some common barriers to AAC use for speaking autistic adults?

Several barriers may prevent AAC use. These include the following:

- Knowledge of AAC options

- Attitudes about who AAC is useful for

- Beliefs that the use of AAC should be decreased if a person can speak

- Cost of AAC devices and applications.

What are our recommendations to autistic adults who might use AAC and their supporters?

- Support autistics in defining their own communication needs, regardless of speech.

- Evaluate communication goals and match them with system features when making decisions about AAC.

- Prioritize all communication, not just speech.

- Explore a variety of options to support communication.

- Explore low- and no-cost options to support communication.

What are some research questions that still need to be answered?

- What communication strategies do autistic adults see as effective?

- What AAC strategies are being used, in what environments, and by whom?

- What prevents effective AAC use?

- How can AAC specialists and autistic adults best collaborate to promote and evaluate AAC use?

- How can communication supports be designed to better meet the needs of autistic adults?

\section{Introduction}

A UGMENTATIVE AND ALTERNATIVE communication (AAC) addresses the needs of people with speech and communication disabilities using a variety of techniques and tools. These tools include communication boards, speech generating devices, manual signs, and other electronic and nonelectronic supports. AAC can both support existing speech and serve as an alternative to speech. ${ }^{1}$ At its most inclusive, AAC covers all the ways people communicate without, or in addition to, verbal speech, including methods used by people with typical speech. ${ }^{2}$

There is significant research into the use of AAC strategies for autistic people. ${ }^{3}$ AAC can support autistic people's communication in a variety of contexts. ${ }^{4-6}$ Several groups work on research, policy, practice, and education regarding AAC. One such group is the National Joint Committee for the Communication Needs of Persons with Severe Disabilities (NJC), which focuses on communication support needs related to intellectual disabilities. ${ }^{7}$ The NJC's resources include the Communication Bill of Rights, which asserts that everyone has the right to affect their life through communication. It then discusses specific communication rights relevant to people with communication disabilities, including rights to social interaction, making requests, choosing between meaningful options, rejecting offers, and sharing opinions, comments, and feelings. If a person requires $\mathrm{AAC}$ supports or strategies to ensure those rights, they have the right to functioning AAC at all times. ${ }^{8}$

Autistic people of all ages and abilities use AAC. ${ }^{3}$ With the increased availability of tablets, mobile applications, and information about AAC, many schools and families provide children with AAC applications. ${ }^{9}$ Companies that design and market AAC tools, such as Speak for Yourself ${ }^{10}$ and AssistiveWare, ${ }^{11}$ often have websites with information about their tools, AAC in general, and user stories. These sites can help potential customers decide if a given tool will meet their needs. Parents or professionals may also create web pages, such as PrAACtical AAC, where updates about discounts on AAC applications, ${ }^{12}$ tools for assessing needs and implementing AAC, ${ }^{13}$ and references to relevant peer-reviewed literature are available. ${ }^{14}$

In addition to families introducing AAC supports for their children, autistic adults may also acquire AAC supports for their own use. ${ }^{15-17}$ However, research into AAC use for autistic people, like other autism research, has typically focused on children, so there is little academic work on AAC use by autistic adults. ${ }^{4}$

AAC research has also typically focused on people without "functional speech," which can be defined as the ability to use at least six words or multiword phrases. ${ }^{5}$ This focus is not unique to autism, ${ }^{18}$ although there are some exceptions. Some studies on adult AAC users with other disabilities note that some participants also speak, ${ }^{19}$ and a recent survey of AAC use indicates that many, and perhaps most, adult AAC users make use of oral speech as well as AAC. ${ }^{20}$ There is also record of five autistic students with functional speech benefiting from AAC interventions. ${ }^{6}$ Despite this, estimates of how many autistic people will require AAC are determined by statistics of how many autistic people have functional speech. ${ }^{5}$

Combining the tendency to focus on autistic children, rather than adults, with the tendency to focus on people who cannot use speech, there is little evidence of work on AAC for autistic adults with some oral speech abilities. However, autistic adults who can speak still experience communication difficulties. ${ }^{21-24}$ We also know that some autistic adults use AAC to address these barriers, as they discuss their AAC use publicly. ${ }^{15,16,21,22}$ The first author is a speaking autistic person who uses AAC some of the time and discusses their own AAC use publicly. While seeking information relevant to their own AAC use helped inspire the current project, this article does not discuss their own AAC use. The second author is an education professional in the field of special education 
for more than 40 years, a large part of which was working with adults with disabilities (including autistic adults) in a community college setting, and later in a university-affiliated program focused on developmental disabilities. She has worked with assistive technology, including AAC, since 1985. In the remainder of this article, we seek to explain some communication difficulties autistic adults address with AAC, AAC strategies that autistic adults use, and barriers to AAC use. We then provide recommendations for practice and research regarding autistic adults who may benefit from AAC.

\section{Autistic Adults with Oral Speech and AAC Use}

\section{Speech for autistic adults}

Autistic people have a mix of context-related strengths and weaknesses in language, communication, and social interaction. ${ }^{23}$ Some autistic people do not speak at all, or do not use speech to communicate. Autistic people who use speech may experience intermittent, unreliable, and/or insufficient speech. A person with intermittent speech can speak sometimes, but not always, and a person with unreliable speech may say things that do not match their preferences or intended meaning. ${ }^{24}$ We use these definitions and add insufficient speech. In insufficient speech, a person's oral speech agrees with their knowledge and beliefs, but does not fully meet their communication needs. That is, the person can speak orally and accurately, but not completely - they cannot always communicate everything one wishes to, using oral speech alone. In all three cases, a person could be considered to have functional speech but requires additional communication supports or strategies to access their full communication rights.

There do not seem to be formal studies of intermittent speech in autistic adults that consider intermittent speech as potentially distinct from selective mutism. While there are some outward similarities between the intermittent speech described by many autistic people and selective mutism described in the Diagnostic and Statistical Manual of Mental Disorders (DSM), ${ }^{25}$ there are differences between the two. Selective mutism is a "consistent failure to speak in certain social situations (e.g., school) where there is a natural expectation of speaking." ${ }^{25}$ Intermittent speech does not always follow this pattern. Illness and overall stress can cause this temporary inability to speak, ${ }^{24}$ as can sensory overload ${ }^{26}$ and meltdowns. ${ }^{27}$ Co-occurring conditions such as migraines ${ }^{17}$ and epilepsy ${ }^{28}$ may also cause an autistic person to be unable to speak. These factors appear independently from most social environments, or nearly so.

To put it simply, "Even verbal autistic people are likely to have difficulty being verbal all the time."26(p.54) Speech may be a limited resource to ration to avoid running out in an emergency, ${ }^{29}$ which, again, differs from consistently not speaking in certain social situations. In this case, a person may choose to write in one situation so they can still speak if they later find they need to call emergency services. ${ }^{29}$ Another difference between the intermittent speech described by autistic adults and selective mutism described in the DSM is that selective mutism is an anxiety disorder. ${ }^{25}$ Anxiety is not the only reason autistic adults find themselves unable to speak, ${ }^{30}$ and may not relate to an autistic adult's intermittent speech. ${ }^{29}$ Finally, selective mutism is not an appropriate diagnosis when a communication disability or autism explains the lack of speech. ${ }^{25}$
Unreliable speech occurs when a person's speech and intended meaning disagree. The unintentional use of an interaction script, such as a person responding to "How are you doing?" with "Fine, thanks, and you?" whether or not one is actually fine, is one form of unreliable speech. ${ }^{31}$ Note that it is the unintended use of a script that does not match current needs, not the script itself, that makes this speech unreliable. This mismatch between speech output and communication needs leads to communication breakdowns, ${ }^{32}$ with sometimes significant consequences. Imagine using the "I'm fine, how are you?" script at the doctor's office! ${ }^{31}$ For others, the reliability issue may be one of precision, where a person has to choose between speaking something close to the desired meaning, or typing exactly what is meant. ${ }^{16}$ In this case, slowing down may be an advantage, ${ }^{33}$ because it provides the opportunity to recognize and avoid unintended meanings.

Regarding insufficient speech, it is important to note that different types of speech may pose different levels of difficulty. Talking about past and current feelings or experiences may be harder than transmitting factual information. ${ }^{21,34,35} \mathrm{It}$ may also be difficult to ask for help or explain what is going on under stress. ${ }^{33}$ Socialization is yet another area where the expectation of oral speech can be a barrier. ${ }^{16,28}$ While some types of speech (e.g., speech with novel or emotional content) are often described as more difficult than others (e.g., scripted speech or speech that conveys largely factual content), these patterns vary between speakers. ${ }^{15,22,24,31,35}$

\section{AAC strategies adopted by speaking autistic adults}

Autistic AAC users who use speech, or who at some point used speech, reference a variety of technologies and strategies. The three methods most commonly referenced seem to be (1) free or low-cost tools that are not specific to AAC, (2) mobile applications designed for communication support, and (3) signed languages such as American Sign Language (ASL). ${ }^{15,16,22}$

For some people, and in some situations, simply writing or typing is sufficient, without needing an application that is specifically designed for AAC. Typed Words, Loud Voices ${ }^{22}$ describes itself as an anthology by people who type to communicate, without requiring that the typing occurs in a context that would be considered AAC for those without speech disabilities. For example, autistic communities have grown from e-mail lists where communication is already text based. ${ }^{26}$ Online chat rooms and unlimited texting plans have similarly improved social communication and allowed friendships to grow. ${ }^{28}$ Information and communication technologies have provided important options for a variety of AAC users. ${ }^{36-38}$ For in-person interactions, writing or typing in advance and then showing the results to conversational partners is one option. ${ }^{15}$ Writing in the moment is another. ${ }^{29}$

It is also possible to carry or wear messages with predetermined meanings and use them as needed. Communication cards may be a communication system, or supplement other communication systems. ${ }^{39}$ Worn objects, such as cards or badges, have also been used to support communication in autistic spaces for some time. In particular, color-coded communication badges are common at conferences and conventions to indicate attendees' interaction preferences. ${ }^{26}$ Some have even suggested that the content on T-shirts is a form of AAC. ${ }^{40}$

In addition, autistic adults may use mobile applications designed for AAC. These applications include text-based 
applications such as Emergency Chat ${ }^{\mathrm{TM}},{ }^{27}$ Flip Writer $^{\mathrm{TM}}$, and Proloquo4Text ${ }^{\mathrm{TM}}{ }^{15}$ They also include applications with picture supports such as Sono Flex Lite ${ }^{\mathrm{TM}} .{ }^{41}$ Mobile applications have the advantage of portability and may be useful in situations where larger AAC systems are impractical. ${ }^{42}$ For some, these high-technology options are the backup AAC option, rather than the preferred one. ${ }^{39}$ However, their use is common when they are affordable. Some autistic adults even design their own applications with AAC functions, including Emergency Chat ${ }^{\mathrm{TM}},{ }^{27}$ the Autistic Space $\mathrm{Kit}^{\mathrm{TM}}{ }^{43}$ and individual projects. ${ }^{16}$

Others report learning a signed language, such as ASL, for the purpose of communicating when speech is not possible. ${ }^{39,44,45}$ One limitation of ASL is that if a signer's communication partner does not know ASL, then an interpreter is required. For this reason, autistic adults who learn ASL as an alternative to speaking may need other AAC strategies as well. $^{39,44,45}$ For example, one person who uses ASL as his preferred communication method has a backup text to speech system, and sometimes wears communication cards with emergency information and a statement that a neurological disability affects the gait. ${ }^{39}$ Another uses Verbally ${ }^{\mathrm{TM}}$ on an iPad as well as ASL. ${ }^{44}$ Yet another mixes writing, ASL, voice recorder applications, text to speech applications, and speech with sometimes nonstandard syntax. ${ }^{45}$ The specific combinations of communication strategies used vary, but combining communication strategies is common, as is the use of alternative communication strategies alongside speech.

In addition to combinations of strategies discussed above, people may also gesture or make nonspeech sounds to express affirmations, refutations, refusals, or requests for more time. This can be particularly effective with familiar communication partners, who may be better able to understand idiosyncratic gestures or vocalizations. Some people may bring familiar communication partners with them under certain circumstances for this reason. ${ }^{46}$ In general, combining communication strategies can increase flexibility and allow for successful communication in varied environments. ${ }^{47}$

\section{Barriers to AAC use faced by speaking autistic adults}

Autistic adults who may benefit from AAC supports commonly face knowledge barriers to doing so. They, like their parents, may be unaware of AAC options ${ }^{48}$ or mistakenly believe AAC is only for people who cannot speak at all. ${ }^{49,50}$ Even those adults who were identified and received communication support in childhood may have missed out on the current wave of technologies supporting AAC, as is the case for many adults with developmental disabilities. ${ }^{51}$ Even those who are aware of AAC may not be aware of relevant AAC support options, ${ }^{15}$ especially given the lack of research on AAC for autistic adults. ${ }^{4}$ Specific skills may also be required to effectively use relevant $\mathrm{AAC}$ options, or to communicate under restrictions inherent to certain AAC systems. $^{52}$

In addition, many autistic adults are either misdiagnosed or lack a diagnosis entirely. ${ }^{53,54}$ This is particularly relevant as AAC service provision has often been tied to diagnosis. ${ }^{5}$ For this reason, many autistic adults do not have access to professional expertise in matching their communication abilities and goals with the features of AAC systems and strategies, as ideally occurs in AAC evaluation. ${ }^{9,55}$
Another major barrier to the acquisition and use of AAC strategies is cost. As autistic adults considered to have functional speech are not receiving insurance funding for the acquisition of speech generating devices, the technology to support any AAC strategies must be purchased out of pocket. However, autistic adults are frequently under- or unemployed, ${ }^{23}$ which makes self-funding applications or devices difficult. This may partially explain the adoption of free and low-cost strategies such as texting in face-to-face communication, free or inexpensive mobile applications, and penand-paper or gesture-based solutions. It may also partially explain decisions to program one's own AAC applications.

Even for autistic adults with the knowledge and financial means to implement appropriate AAC supports, attitudinal barriers remain an issue. People who could benefit from communication supports may be afraid to use them because they do not match popular ideas of AAC users, and may have been told explicitly that using communication aids would therefore be disrespectful. ${ }^{50}$ People may also fail to recognize communication breakdowns or the utility of AAC supports in addressing these breakdowns due to a focus on existing speech. $^{21,56}$

Parents or professionals may also mistakenly attribute intermittent, unreliable, or insufficient speech to anxiety, ${ }^{29}$ and therefore presume a reduction in anxiety will thus reduce the need for AAC supports. ${ }^{30}$ When anxiety is a communication barrier, it is often anxiety about other communication barriers, including these attitudinal barriers. Both accusations of feigning one's need for $\mathrm{AAC}^{49}$ and anxiety about being perceived as faking ${ }^{56}$ can impede communication. Similarly, worries about being denied access to communication supports by professionals who do not support AAC use may be a barrier to AAC use. ${ }^{30}$

Another barrier comes from the presumption that reducing AAC use is, or should be, a priority. ${ }^{30}$ This may tie in with indistinguishability from one's peers and the appearance of neurotypical communication as a goal, ${ }^{57}$ or with the assumption that if an autistic person is "permitted to type," they will then not speak. ${ }^{21}$ While the opposite is more likely to be true, ${ }^{3}$ the concern is common, and tends to come with the assumption that AAC is a "last resort" only to be used when speech has already failed. ${ }^{58}$ Whether or not a person would choose to speak less given access to AAC, this is a poor reason to deny AAC access. The assumption that AAC will impede speech, in combination with the prioritization of speech over other forms of communication, makes it harder for speaking autistic adults to make full use of AAC strategies.

\section{Recommendations}

The use of AAC strategies to support communication in autistic adults has received limited attention to date ${ }^{4}$ and is not often considered in the presence of functional speech. ${ }^{48}$ However, autistic adults who can speak describe significant benefits from the use of AAC strategies, ${ }^{15-17,21,22}$ sometimes designing their own applications. ${ }^{27,43}$ Based on a combination of AAC practices and the descriptions of autistic AAC users, we provide suggestions for autistic adults who use AAC or wish to use AAC, a variety of people supporting such adults, and researchers looking to do further work on speech and AAC for autistic adults. 


\section{Practice recommendations for (potential) AAC users and those supporting them}

In all cases, we consider (potential) AAC users as primary experts in their needs. ${ }^{59}$ Many different people support (potential) AAC users, ideally including family members, friends, a wide variety of professionals who work with autistic adults, and others involved in a person's day to day life. While recommendations related to the AAC evaluation may be limited to a smaller group involved in formal decisionmaking, attitudinal choices such as prioritizing communication over speech and supporting a variety of communication strategies may apply to all communication partners, including professionals from many fields.

Consider broader communication needs, including the fully revised Communication Bill of Rights, ${ }^{8}$ not just the presence or apparent fluency of functional speech, when deciding whether to use AAC supports. The ability to say at least six words or short phrases counts as functional speech. ${ }^{5}$ However, this is not sufficient for full participation in many contexts. The Communication Bill of Rights, which holds that all people have the right to affect their conditions through communication, sets a higher bar. It states people have the right to make requests, to reject offered options, and to express opinions, preferences, and feelings. ${ }^{8}$ As shown by the descriptions of several autistic adults, neither functional speech nor fluent-seeming speech implies these abilities. ${ }^{22}$ Even adults whose parents describe them as able to communicate their needs experience frequent communication breakdowns and may therefore benefit from AAC supports. ${ }^{48}$ Given a need for AAC, the Communication Bill of Rights declares the right to functioning AAC at all times. ${ }^{8}$ All persons involved in decisions regarding communication supports should thus consider broader communication needs.

Communication, not oral speech, should be the priority. People supporting potential AAC users may express concerns about AAC interfering with speech or language development. This is an especially common concern for people with some speech abilities. ${ }^{42}$ While AAC use does not impede speech, ${ }^{3,57}$ overall communication should be prioritized regardless of personal decisions made about the use of speech. Some autistic adults use AAC even when they can technically speak, as speech cannot meet all their communication needs, ${ }^{22,49}$ or speech is simply less effective or more effortful than alternatives. ${ }^{50}$ In these cases, we must recognize that communication ease and effectiveness differ from, and may conflict with, the appearance of neurotypicality. ${ }^{57}$ That is, a focus on typical speech may impede communication by neglecting the impact of communication breakdowns and the utility of communication supports. In any case, people with typical speech are frequently able to choose communication methods by personal preference, such as preferring texting to calling. AAC users and potential AAC users should have the same communication rights. As such, the use of AAC should be normalized and encouraged whenever it can support communication, including in the presence of speech.

Evaluate communication abilities and goals as well as system features when selecting AAC strategies. In general, it is important to consider communication abilities and goals when determining what an AAC user will need from the system. These needs should then be matched with the supports a given system or strategy provides. ${ }^{55}$ This remains important when considering mobile applications. ${ }^{9}$ Otherwise, there may be mismatches between individual needs and system design, reducing the effectiveness and use of communication supports. ${ }^{15,16,41}$

For example, a person who uses AAC supports in environments where space is limited is likely to prefer systems on mobile platforms or small low-technology supports that can be carried easily. A rapid typist may prefer a text-based system and put less priority on saved words or phrases. Someone who has difficulty reading or writing at times, or who is simply a visual thinker, may prefer a system with more picture symbols. The longer a person goes without access to electrical outlets, the more likely they are to require a long battery life or a low- or no-technology solution.

Make use of information and communication technologies. The advantages of asynchronous communication via texting $^{36}$ and over the Internet ${ }^{37}$ for AAC users have been discussed for as long as the technologies have been available. Autistic adults, whether or not they are typically considered AAC users, have similarly made frequent use of the Internet ${ }^{26}$ and texting. ${ }^{28}$ Just as the use of information and communication technologies is supported for other AAC users, ${ }^{38}$ it should be supported for autistic adults.

Remember the "augmentative" part of AAC. Some autistic people report using strategies to support existing speech, including visual supports. This is in line with the use of augmentative strategies for people who speak ${ }^{18}$ and the use of visual communication supports for autistic people. ${ }^{42}$ Others may type out statements before reading them aloud. The use of these strategies may be compared with similar strategies used by nondisabled adults before and during presentations, and should be normalized and encouraged in any contexts where they support communication.

Use, and support the use of, a variety of communication methods and supports. Autistic AAC users describe many communication methods and supports, varying with their situation and specific needs. ${ }^{15,16,22}$ Using several AAC strategies increases communicative flexibility and can allow for successful communication in varied environments. ${ }^{47}$ It also parallels the variety of communication strategies used by typical speakers in daily communication, and should be supported.

In addition, exposing AAC users to many different communication support options, including low- and no-technology options, helps ensure they encounter the options that will work best for them. Exposure, however, does not mean pushing AAC users toward or away from specific options. Certain technologies, including text to speech, may not be accessible for all users. ${ }^{29}$ Individual choices to reject even popular AAC options, such as speech generation or picture-based systems, must be respected.

\section{Recommendations for future research}

The above suggestions for potential AAC users and those supporting them are based on a combination of strategies that speaking autistic adults have found effective in supporting communication and AAC research on other disabled populations. This includes research addressing autistic children and nonautistic people who can speak. However, the authors only found one study that specifically noted AAC technology 
provision for autistic children who could speak, ${ }^{6}$ and there was no indication of oral speech abilities for the four adult participants found in a systematic review of AAC intervention research for autistic adolescents and adults. ${ }^{4}$ Mainstream AAC supports designed for autistic adults, speaking or not, are also lacking - the applications designed by autistic adults have reached a limited audience thus far, and are typically based on the specific needs of the single users designing them. While individualized designs are ideal for their specific users, and may in some cases prove useful for AAC development as a whole, implementing them for others as-is does not provide all users with appropriate supports. Further work in these areas is needed. The below areas may be ideal topics for collaboration between autistic AAC users and researchers, both academic and clinical, in their respective fields.

It would be useful to know how well various communication strategies are meeting the needs of autistic adults with functional speech. Typing is often used to support communication $^{22}$ and communication can be a concern in many contexts. $^{21-24,26}$ A more specific understanding of communication support needs could be achieved with an understanding of required communicative functions, ${ }^{8}$ specific skill sets needed when using AAC, ${ }^{52}$ and the patterns of communication differences described by autistic adults to show areas of potential need.

It would also be beneficial to better understand if, when, and how autistic adults are supporting their communication with $A A C$ strategies. A small sample of autistic adults who are sufficiently interested in AAC to write publicly about it provide current information on AAC use. ${ }^{15-17,21,22,27,30,39-41,43,49,50}$ However, this sample may or may not prove representative. An understanding of how communication needs are already being addressed with AAC will improve recommendations for people pursuing AAC supports and provide a baseline for research on improving AAC supports for autistic adults.

A better understanding of barriers to AAC use for autistic adults could be used to address these barriers. As discussed above, barriers to AAC use for autistic adults may include lack of awareness of relevant options, ${ }^{48}$ lack of access to services, ${ }^{5}$ focus on existing speech, ${ }^{21,58} \mathrm{cost}^{23}$ and social stigma or misconceptions about who AAC supports are for. $^{21,49,50}$ It is not yet clear, however, what other barriers may be present, which barriers have the greatest effects, or how to address barriers to AAC use by autistic adults.

It is not known how intermittent speech that is not explained by selective mutism works in autistic adults. As noted, when discussing intermittent speech, this phenomenon does not strictly fit within the definition of selective mutism. This, however, is merely a partial understanding of what intermittent speech in autistic adults may not be. This could be a topic of further neurological, psychological, and/or speech and language/pathological research.

Finally, it would be of use to understand the potential role of AAC specialists in providing these supports. At present, speaking autistic adults who use AAC rely on peer knowledge, work on AAC with limited application to their own situations, and trial and error to select and effectively use their systems. It would, therefore, be useful for AAC users and professionals to collaboratively develop evaluation and teaching strategies that respect both the individual and community knowledge and skills of potential AAC users, while making use of professional expertise.

\section{Acknowledgments}

We thank Sam Harvey, Anna Williams, Derek Burrow, Dr. Nick Chown, and all of our reviewers for their suggestions on the article. We also thank all the autistic people interested in AAC for publicly sharing both their expertise on the subject and their personal experiences. It is thanks to them that we have anything to cite on the topic of speaking autistic adults who use AAC and that Alyssa, too, makes use of AAC part time.

\section{Authors' Contributions}

As first author, Alyssa Hillary Zisk was involved in formulating the research goals, found sources, created recommendations, and wrote and edited the article. Dr. Elizabeth Dalton was involved in formulating research goals and questions, revised the article, and played a supervisory role throughout the project. Both authors approve the final version. This work has not been submitted elsewhere or published elsewhere. It is not under review anywhere else. It is also not on a preprint server.

\section{Author Disclosure Statement}

No competing financial interests exist.

\section{References}

1. American Speech-Language-Hearing Association. Augmentative and alternative communication. https://www.asha. org/Practice-Portal/Professional-Issues/Augmentative-andAlternative-Communication Accessed December 10, 2017.

2. American Speech-Language-Hearing Association. Augmentative and alternative communication (AAC). https:// www.asha.org/public/speech/disorders/AAC Accessed December 10, 2017.

3. Mirenda P. Augmentative and alternative communication. In: Volkmar FR, ed. Encyclopedia of Autism Spectrum Disorders. New York, NY: Springer-Verlag; 2013;328-333.

4. Holyfield C, Drager KD, Kremkow JM, Light J. Systematic review of AAC intervention research for adolescents and adults with autism spectrum disorder. Augment Altern Commun. 2017;33(4):201-212.

5. Mirenda P. Autism spectrum disorder: Past, present, and future. Perspect Augment Altern Commun. 2013;22(3): 131-138.

6. Mirenda P, Wilk D, Carson P. A retrospective analysis of technology use patterns of students with autism over a fiveyear period. J Spec Educ Technol. 2000;15(3):5-16.

7. National Joint Committee for the Communication Needs of Persons with Severe Disabilities (NJC.) History of the National Joint Committee. American Speech-LanguageHearing Association. https://www.asha.org/NJC/history Accessed October 8, 2018.

8. Brady NC, Bruce S, Goldman A, et al. Communication services and supports for individuals with severe disabilities: Guidance for assessment and intervention. Am J Intellect Dev Disabil. 2016;121(2):121-138.

9. Farrall J. AAC apps and ASD: Giving voice to good practice. Perspect Augment Altern Commun. 2013;22(3):157-163.

10. Speak for Yourself AAC. Www.speakforyourself.org Accessed October 8, 2018.

11. AssistiveWare. www.assistiveware.com Accessed October 8, 2018. 
12. Zangari C. PrAACtical Alert: AAC App Discounts. PrAACtical AAC. Published March 22, 2018. http:// praacticalaac.org/praactical/praactical-alert-aac-app-discounts Accessed October 8, 2018.

13. Zangari C. Toolbox. PrAACtical AAC. http://praacticalaac .org/toolbox Accessed October 8, 2018.

14. Zangari C. 5 References That Support the Use of AAC for People with ASD. PrAACtical AAC. Published February 1, 2013. http://praacticalaac.org/praactical/5-references-thatsupport-the-use-of-aac-for-people-with-asd Accessed October 8, 2018.

15. Theoriesofminds, ed. Jul 2, 2017: AAC (augmentative and alternative communication) (transcript). Autchat. Published July 7, 2017. http://autchat.com/jul-2-2017-aac-augmentativeand-alternative-communication-transcript Accessed December 10, 2017.

16. Theoriesofminds, ed. Aug 13, 2017: Useful Apps. Autchat. Published August 20, 2017. http://autchat.com/aug-132017-useful-apps Accessed December 10, 2017.

17. When my speech shorts out I use this program. AssistiveWare. Published July 9, 2015. http://www.assistiveware.com/ when-my-speech-shorts-out-i-use-program Accessed December 10, 2017.

18. Hanson E. My client talks! Do I still need to consider AAC in my treatment planning? Speech supplementation strategies: AAC for clients who talk!. Perspect Augment Altern Commun. 2014;23(3):124-131.

19. Rackensperger T, Krezman C, Mcnaughton D, Williams MB, D' silva K. "When I first got it, I wanted to throw it off a cliff": The challenges and benefits of learning AAC technologies as described by adults who use AAC. Augment Altern Commun. 2005;21(3):165-186.

20. Niemeijer D. The state of AAC in English-speaking countries: First results from the survey. AssistiveWare. November 15, 2015. www.assistiveware.com/state-aac-english-speakingcountries-first-results-survey Accessed December 10, 2017.

21. Wayman L, Rothschild C, Cummings C. But You Can Talk! Alternative Communication for Verbal Individuals with ASD. Presented at 49th Annual Autism Society National Conference; July 12-15, 2017; Milwaukee, WI. https://asa.confex.com/asa/2017/webprogramarchives/ Session9756.html Accessed December 10, 2017.

22. Sequenzia A, Grace EJ, eds. Typed Words, Loud Voices. Fort Worth, TX: Autonomous Press; 2015.

23. Robertson S. Neurodiversity, quality of life, and autistic adults: Shifting research and professional focuses onto reallife challenges. Disabil Stud Q. 2010;30(1). http://doi.org/ 10.18061/dsq.v30i1.1069.

24. Sparrow M. Coping with a Crisis When You Have Unreliable or Intermittent Speech. Thinking Persons Guide to Autism. Published November 13, 2017. http://www .thinkingautismguide.com/2017/11/coping-with-crisis-whenyou-have.html Accessed December 10, 2017.

25. American Psychiatric Association. Anxiety disorders. In: Diagnostic and Statistical Manual of Mental Disorders, 5th ed. Washington, DC: American Psychiatric Association; 2013. https://dsm.psychiatryonline.org/doi/full/10.1176/appi.books $.9780890425596 . d s m 05$

26. Sinclair J. Autism Network International: The development of a community and its culture. In Bascom J, ed. Loud Hands: Autistic People Speaking. Washington, DC: The Autistic Press; 2012;22-70.

27. Murphy J. Having a meltdown? Use the emergency chat app for help. Geek club books: Autism storytelling. Pub- lished August 6, 2015. https://geekclubbooks.com/2015/08/ meltdown-emergency-chat-app-help Accessed December 10, 2017.

28. Sibley KA. My cat comes when my tablet calls him. In: Sequenzia A, Grace EJ, eds. Typed Words, Loud Voices. Fort Worth, TX: Autonomous Press; 2015;60-62.

29. Allen B. Notes on not speaking. In: Sequenzia A, Grace EJ, eds. Typed Words, Loud Voices. Fort Worth, TX: Autonomous Press; 2015;29-30.

30. Baggs A. Autism, speech, and assistive technology. In: Bascom J, ed. Loud Hands: Autistic People Speaking. Washington, DC: The Autistic Press; 2012;320-323.

31. Asasumasu K. If you don't use your words you won't be indistinguishable. Radical Neurodivergence Speaking. Published October 4, 2013. https://timetolisten.blogspot.com/ 2013/10/if-you-dont-use-your-words-you-wont-be.html Accessed October 8, 2018.

32. Kimmel A. Interview. In: Sequenzia A, Grace EJ, eds. Typed Words, Loud Voices. Fort Worth, TX: Autonomous Press; 2015;29-30.

33. von Woerkom A. June 16, 2002. In: Sequenzia A, Grace EJ, eds. Typed Words, Loud Voices. Fort Worth, TX: Autonomous Press; 2015;30-31.

34. Kim C. A barrier of sound, not of feeling. In: Sequenzia A, Grace EJ, eds. Typed Words, Loud Voices. Fort Worth, TX: Autonomous Press; 2015;17-20.

35. Mamautistic. Time, Mutism, and Levels of Communication. June 13, 2017. https://mamautistic.wordpress.com/ 2017/06/13/time-mutism-and-levels-of-communication Accessed December 10, 2017.

36. Williams M, Beukelman D, Ullman C. (2012). AAC text messaging. Perspect Augment Altern Commun. 2012;21(2): 56-59.

37. Williams M. The internet and AAC. Alternatively Speaking. 1995;2(3):1-6.

38. Bryen DN, Chung Y, Lever S. What you might not find in a typical transition plan! Some important lessons from adults who rely on augmentative and alternative communication. Perspect Augment Altern Commun. 2010;19(2):32-40.

39. Sedgewick MZ. The rugged beauty of creating a neuroqueer space. In Ryskamp D, Harvey S, eds. Spoon Knife 2: Test Chamber. Fort Worth, TX: Neuroqueer Press; 2017;140-151.

40. @endeverstar. HOLY SHIT TSHIRTS ARE AAC THIS EXPLAINS SO MUCH ABT MY WARDROBE $\hookrightarrow$ homo qui vixit $\hookrightarrow$ added, Julia Maureen @ onyourownside Replying to @endeverstar they're aac. https://twitter.com/ endeverstar/status/934127410546483200. Posted November 24, 2017.

41. @ derekthebard. This is one of the AAC apps I use, Sono Flex Lite by @TobiiTechnology It was actually my first AAC app. I find its interface a bit too slow for my needs (to be fair its not really made for me), so I mostly use it for preset phrases (food orders, basic pleasentries, etc.) https:// twitter.com/derekthebard/status/940738897402671104. Posted December 12, 2017.

42. Sennott S, Bowker A. Autism, AAC, and Proloquo2Go. Perspect Augment Altern Commun. 2009;18(4):137-145.

43. Murray D, Brook K, Brook K. ASK: Autistic space kit: Autistic empowerment for the 21st century. http:// www.autisticspacekit.co.uk/pdf/poster.pdf Accessed December 10, 2017.

44. Jones SR. The song of life unfolding. In: Sequenzia A, Grace EJ, eds. Typed Words, Loud Voices. Fort Worth, TX: Autonomous Press; 2015;119-124. 
45. Anonymous. My many communication systems. In: Sequenzia A, Grace EJ, eds. Typed Words, Loud Voices. Fort Worth, TX: Autonomous Press; 2015;90-96.

46. Asasumasu K. Allistic to Neurodivergent K Interpreters are GOOD. We Are Like Your Child. Published July 13, 2013. https://wearelikeyourchild.blogspot.com/2013/07/ allistic-to-neurodivergent-k.html Accessed October 8, 2018.

47. Sigafoos J, Drasgow E. Conditional use of aided and unaided AAC: A review and clinical case demonstration. Focus Autism Other Dev Disabl. 2001;16(3):152-161.

48. Hines M, Balandin S, Togher L. Communication and AAC in the lives of adults with autism: the stories of their older parents. Augment Altern Commun. 2011; 27(4):256-266.

49. Non Speaking Not Silent. Comming out, telling friends and family you communicate using AAC. AAC Apps, Service Dogs, and Autistic Life Things. Published November 1, 2015. http://nonspeakingnotsilent.blogspot.ca/2015/10/ comming-out-telling-friends-and-family.html Accessed December 26, 2017.

50. Baggs M. Communication page I used to handle that invasive woman I met. Ballastexistenz. Published June 25, 2012. https://ballastexistenz.wordpress.com/2012/06/25/ communication-page-i-used-to-handle-that-invasive-womani-met Accessed October 8, 2018.

51. Hughes D. Editorial: The evolving profile of AT and AAC Users. Perspect Augment Altern Commun. 2014; 23(3):115-116.

52. Light J. Toward a definition of communicative competence for individuals using augmentative and alternative communication systems. Augment Altern Commun. 1989;5(2): 137-144.

53. Lewis LF. Exploring the experience of self-diagnosis of autism spectrum disorder in adults. Arch Psychiatr Nurs. 2016;30(5), 575-580.
54. Lewis LF. Realizing a diagnosis of autism spectrum disorder as an adult. Int J Ment Health Nurs. 2016;25(4), 346-354.

55. Beukelman DR, Mirenda P. Augmentative and Alternative Communication: Supporting Children and Adults with Complex Communication Needs, 4th ed. Baltimore, MD: Paul H. Brookes Publishing Co.; 2013.

56.@ @rsocialskills. @@yes_thattoo I have bad anxiety about being perceived as faking; can break communication that would otherwise work. \#ussaachat. https://twitter.com/ rsocialskills/status/819717710292590593. Posted January 12, 2017.

57. Alexis D. On functioning and "functioning." Autistic Academic. June 2, 2015. https://autisticacademic.com/2015/ 06/02/on-functioning-and-functioning Accessed December 26, 2017.

58. Romski M, Sevcik RA. Augmentative communication and early intervention: Myths and realities. Infants Young Child. 2005;18(3):174-185.

59. Elmore K, Meloncon L, James CA. Embracing interdependence: Technology developers, autistic users, and technical communicators. In: Meloncon L, ed. Rhetorical Accessability: At the Intersection of Technical Communication and Disability Studies. New York, NY: Routledge; 2014;15-38.

Address correspondence to: Alyssa Hillary Zisk, MS (They/them/theirs) Interdisciplinary Neuroscience Program University of Rhode Island 67 Upper College Road Kingston, RI 02881

E-mail: alyssazisk@uri.edu 